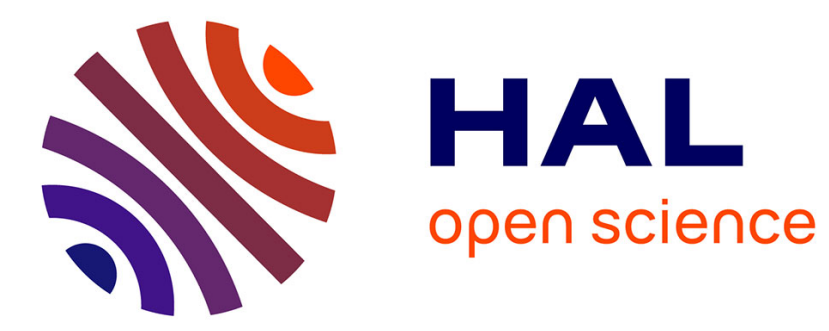

\title{
Situations d'incertitude et rumeurs
}

Véronique Campion-Vincent

\section{- To cite this version:}

Véronique Campion-Vincent. Situations d'incertitude et rumeurs. Communications, 1990, 52 (1), pp.51 - 60. 10.3406/comm.1990.1782 . halshs-01697382

\section{HAL Id: halshs-01697382 \\ https://shs.hal.science/halshs-01697382}

Submitted on 31 Jan 2018

HAL is a multi-disciplinary open access archive for the deposit and dissemination of scientific research documents, whether they are published or not. The documents may come from teaching and research institutions in France or abroad, or from public or private research centers.
L'archive ouverte pluridisciplinaire HAL, est destinée au dépôt et à la diffusion de documents scientifiques de niveau recherche, publiés ou non, émanant des établissements d'enseignement et de recherche français ou étrangers, des laboratoires publics ou privés. 


\section{Situations d'incertitude et rumeurs}

Véronique Campion-Vincent

\section{Citer ce document / Cite this document :}

Campion-Vincent Véronique. Situations d'incertitude et rumeurs. In: Communications, 52, 1990. Rumeurs et légendes contemporaines. pp. 51-60;

doi : 10.3406/comm.1990.1782

http://www.persee.fr/doc/comm_0588-8018_1990_num_52_1_1782

Document généré le 21/03/2017 
Véronique Campion-Vincent

\section{Situations d'incertitude et rumeurs : disparitions et meurtres d'enfants}

INTRODUCTION

L'influence des événements exceptionnels sur la création collective de rumeurs est bien connue et a été largement étudiée. Toute une littérature a ainsi été consacrée à la description des explosions de rumeurs parmi les groupes traumatisés par des catastrophes naturelles (inondations, tremblements de terre) ou sociales (émeutes, guerres) entraînant un bouleversement radical du cadre quotidien de l'existence (voir Shibutani).

J'ai déjà évoqué (voir Campion-Vincent) les réactions des Parisiens de 1750 confrontés à une série exceptionnelle de disparitions d'enfants. D'une part, actions violentes et émeutes contre la police responsable de la plupart de ces disparitions qui correspondaient à des enlèvements. D'autre part, accusations fantasmatiques actualisant d'anciens motifs légendaires : le roi, malade de la lèpre, pouvant guérir grâce à des bains de sang innocent, aurait été la cause de ces enlèvements destinés à le soigner.

Je m'en tiendrai ici à l'actualité immédiate et tenterai de montrer comment des événements et situations relevant de la chronique, du quotidien, entraînent également une création collective allusive et discrète de rumeurs et de légendes qui peut parfois déboucher également sur une épidémie. Ces événements et situations baignent dans l'incertitude, indiquent une dysfonction de la mécanique sociale, angoissent. Je prendrai pour exemples les cas de disparitions d'enfants, débouchant parfois sur des meurtres.

Je montrerai également comment ces rumeurs sont intégrées dans les stratégies de persuasion et de mobilisation des groupes sociaux les plus concernés par ces phénomènes, les activistes. 


\section{DISPARITIONS}

Réelles ou imaginaires, les disparitions constituent pour les rumeurs un aliment de choix. La collectivité concernée s'émeut particulièrement - et tout un processus rumoral et fantasmatique s'élabore - quand il semble que l'on peut parler de série, d'une succession inquiétante située dans un espace réduit que l'on peut figurer sur la carte par un triangle ou un cercle. Dans un article récent, Kapferer a souligné le caractère fantasmatique de ce processus à la fois médiatique et rumoral de désignation collective du coupable.

Le grand public a des disparitions une perception à la fois intermittente et indirecte. Intermittente : le thème n'est pas une préoccupation constante, mais le devient au hasard de l'actualité. Indirecte : c'est généralement par le relais des médias que l'on connaît ces événements et qu'on y participe.

A propos des disparitions, l'on voit affleurer - même dans les plus respectables des médias - des hypothèses implicites relevant de la littérature feuilletonesque, des adaptations de thèmes légendaires immémoriaux qui voisinent avec les précisions rassurantes et les remarques terre à terre des services officiels.

Les disparitions sont un phénomène, ou plutôt un ensemble de phénomènes, caractérisé par l'incertitude - incertitude que renforce l'absence d'informations fiables aisément accessibles. Plusieurs facteurs doivent être considérés dans l'approche des disparitions, ce sont :

- la difficulté d'évaluation et de mesure;

- la position des officiels;

- les perceptions des activistes;

- les attitudes des médias.

\section{Difficulté de mesure et d'évaluation.}

Le terme de disparition renvoie à la constatation d'une absence immotivée, inexpliquée, à une incertitude sur le sort du disparu. Cette constatation, sa déclaration aux autorités dépendent de la qualité de l'entourage de l'absent.

Une disparition, c'est une situation en évolution qui peut " se fermer " - de façon bénigne ou fatale, à plus ou moins brève échéance ou "rester ouverte", l'incertitude continuant alors à régner.

Une disparition, c'est une situation qui peut avoir diverses expli- 
cations. Sans commencer une analyse minutieuse des causes et paramètres à envisager, sans ouvrir une énumération lassante des cas de figure possibles, nous évoquerons brièvement les principales: fugues, accidents, enlèvements par des proches ou des étrangers. Quelle peut être la signification de chiffres qui amalgament des situations aussi diverses? Faible. Si des statistiques officielles publiées par le ministère de l'Intérieur font état pour 1987 de 18000 personnes majeures recherchées et de 6500 personnes retrouvées, on ne peut guère en conclure que 11500 personnes ont disparu en France cette année-là.

Pour que les chiffres - de recherches d'une part, de recherches ayant abouti de l'autre - puissent permettre d'apprécier l'ampleur des vraies disparitions, tout un travail de pondération et de recherche serait nécessaire - travail qui supposerait une coûteuse mobilisation collective. Plus modestement, je pense prolonger le travail de réflexion et de première approche présenté ici par une étude des chiffres officiels et un contact avec les administrations concernées. En attendant, on ne peut qu'approuver les remarques publiées sur ce sujet en 1982 :

C'est une évidence, il est de plus en plus facile de disparaître dans nos sociétés modernes où le tissu social et familial s'est extraordinairement relâché. [...] Au nom de la liberté individuelle, on néglige gravement les cas des disparitions inquiétantes noyés dans la masse des disparitions volontaires (Patrick Benquet, "Recherches dans l'intérêt des familles ", Le Monde Dimanche, 21 mars 1982).

\section{La position des officiels.}

Les services officiels producteurs de ces chiffres en soulignent les inexactitudes par rapport aux situations dont elles sont le reflet : disparitions non déclarées par un entourage indifférent ou inexistant; retours non signalés; enregistrements des disparitions qui peuvent être répétées et non des personnes... la liste peut être longue.

Faut-il en conclure que les officiels n'affirment rien en la matière? Non, car ils présentent une théorie implicite, un système d'explication rassurant. Les disparitions sont principalement des fugues temporaires, et les cas non résolus correspondent le plus souvent à des départs volontaires. L'augmentation des cas - très forte ces dernières années pour les mineurs (les disparitions de 13- 
18 ans signalées à la police sont passées de 2500 en 1963 à 29000 en 1987) - est liée à des facteurs généraux : émancipation moderne de l'individu (qui affecte spécialement les jeunes); accroissement de la permissivité et de la mobilité; transformation de la cellule familiale.

\section{Les perceptions des activistes.}

Les activistes comprennent plusieurs sous-groupes. Il y a des proches de disparus qui se sont investis dans des tâches d'organisation des recherches, ont lancé des campagnes de mobilisation. On trouve également des travailleurs sociaux et des juristes qui ont été confrontés aux problèmes souvent aigus rencontrés par les proches des disparus et pour lesquels il convient de pallier les insuffisances d'une machinerie administrative perçue et présentée comme indifférente et inhumaine. Il y a des militants pour lesquels il importe de faire reconnaître les disparitions comme un problème social. Certains de ces militants voient dans les disparitions un symptôme des maux qui affectent la civilisation moderne.

En France, l'activisme concernant les disparitions est faible : une poussée en 1982, mais, sept ans plus tard, il y a simplement une ou deux associations, un service télématique (3615 DISP) sur lequel j'ai recensé en août 1989 une soixantaine de cas s'échelonnant de janvier 1988 à mai 1989. Aux États-Unis, il s'agit d'un mouvement important, né à la suite de faits divers dramatiques en 1979 et 1981 , qui mobilise hommes politiques et personnalités médiatiques et a entraîné le vote de législations particulières.

Cependant il est frappant d'observer qu'à un régime d'intensité différent les perceptions des activistes semblent présenter les mêmes caractéristiques.

Les activistes développent une théorie implicite, un système d'explication alarmant. L'accent est mis sur les cas non résolus ou terminés tragiquement. Les chiffres sont extrapolés, grossis de façon à présenter un problème de dimensions majeures.

Fugues, accidents, enlèvements par des proches pour les mineurs sont ignorés, et les activistes suggèrent que l'enlèvement par pulsion sadique ou pour exploitation prostitutionnelle ou pornographique est la cause majeure des disparitions.

Le sociologue américain Best a analysé les affirmations alarmistes des activistes américains en termes d'une stratégie de persuasion, d'une rhétorique. Il a en particulier montré comment, en l'absence de véritables statistiques officielles, la stratégie des activistes est de 
manipuler et grossir les chiffres afin de persuader le public qu'il y a là un problème social majeur. Les chiffres alarmistes lancés en 1981 ont été contestés par les médias en 1985; les activistes ont alors répliqué en élargissant la notion de disparition à toute absence inexpliquée de courte durée.

Mon propos n'est pas de faire de l'angélisme ni de nier la réalité de tels faits. Cependant, les activistes en donnent une version noire largement envahie de fantasmes - comme le montre limportance qu'ils donnent aux enlèvements par des inconnus, ignorant les autres cas de disparitions, qui sont majoritaires.

Leur présentation des disparitions met en valeur leurs aspects les plus angoissants, les plus liés aux thèmes classiques des rumeurs : univers truqué où de noirs desseins se trament; complots maléfiques visant les plus innocents; etc.

En contrepoint, les professionnels de la protection affirment fermement que l'enlèvement est exceptionnel dans les cas de prostitution ou de pornographie:

Les personnes que nous avons retrouvées dans les milieux prostitutionnels, dans les sectes ou autres, étaient toujours consentantes, précise l'inspecteur Dubois de la Neuvième Délégation ("Partis sans laisser d'adresse ", Figaro-Magazine, juillet 1988).

Quelles sont les causes de ces déformations, de cette création collective de rumeurs, de cette reprise de thèmes légendaires?

Au niveau des activistes, on peut citer la préservation du moi : c'est le cas pour les proches des disparus pour lesquels la fugue, le départ volontaire, est particulièrement difficile à admettre. Il y a également le souci de mobiliser les masses, le grand public : grossissement des chiffres et affirmation de la prédominance des enlèvements au hasard, bien plus inquiétants puisqu'ils peuvent concerner chacun d'entre nous. On aurait tort, pourtant, de considérer qu'il y a là propagande et déformation consciente des faits : les perceptions des activistes soint des perceptions alarmées, angoissées, qui justifient leur action.

\section{Les attitudes des médias.}

Cependant, ce sera surtout lors de leur reprise par les médias que ces éléments de rumeur atteindront un large public dont ils orienteront et gauchiront la vision.

Face à ces deux groupes dont les systèmes d'explication rassurants 
(les officiels) et alarmants (les activistes) s'opposent, les médias d'information s'efforcent généralement de présenter les deux systèmes. On trouvera ainsi fréquemment côte à côte des analyses soulignant la prégnance des cas de fugues volontaires dans les disparitions et des présentations des hypothèses alarmistes des activistes. Le journaliste approuve l'un, puis l'autre, sans sembler conscient de la contradiction.

Ce n'est le plus souvent qu'en mineur et de façon allusive que des éléments vraiment fantasmatiques - telles ces snuff-movies où l'on torturerait et tuerait en direct les enlevés pour un public d'amateurs fortunés - apparaissent dans les médias d'information.

Il n'en ira pas de même dans d'autres secteurs des médias:

- Presse populaire récréative destinée avant tout à faire frissonner. On comprend rapidement, en feuilletant ces publications, que leur lecteur ne s'attend pas à y trouver des informations exactes. Ce genre est encore peu répandu en France, par rapport à l'Allemagne ou à l'Angleterre.

- Littérature de masse écrite ou électronique, où l'on trouvera la vraie caisse de résonance des aspects fantasmatiques évoqués plus haut, mais incarnés dans une intrigue narrative.

Une attention particulière doit être portée à ces séries télévisuelles qui ont succédé au roman-feuilleton du quotidien d'il y a 150 ans pour devenir le véhicule majeur de la création littéraire de masse. En particulier à ces séries qui décrivent, avec un réalisme minutieux du cadre physique, la saga de la police dans les grandes villes des États-Unis. Les intrigues romanesques de ces séries développent largement ces thèmes angoissants et fantasmatiques, et il semble probable que - en raison même de leur réalisme - tout un secteur du grand public les confonde de bonne foi avec des reportages ou des documentaires.

\section{LES MEURTRES D'ENFANTS}

C'est cependant surtout quand on sort de l'incertitude pour basculer dans l'horreur que les médias d'information sont concernés par les disparitions. Un sujet mineur débouche alors sur un sujet majeur. C'est ainsi que, à la suite d'une série de meurtres, le mouvement des missing children a pris naissance aux États-Unis.

En France, depuis deux ans, plus d'une vingtaine de meurtres d'enfants et d'adolescents où la pulsion sadique et la rencontre de 
hasard dominent ont créé un climat d'angoisse auquel les médias participent largement.

La série inexpliquée des disparitions de Mourmelon (appelés) n'est par ailleurs pas close. Bien qu'il n'y ait pas eu de preuve de meurtres, de découverte de cadavres, cette série de disparitions est maintenant considérée comme un ensemble de meurtres par les autorités policières et judiciaires. On peut d'ailleurs noter que le refus de l'autorité militaire de considérer ces disparitions comme des meurtres - les familles n'étant pas autorisées à agir en justice a renforcé le processus de rumeurs. Cependant, l'arrestation, en août 1988, d'un suspect correspondant cette fois-ci au profil qu'en dessinait la rumeur n'a pas débouché, un an plus tard, sur une inculpation. Dans d'autres cas - soit de meurtres en série comme le tueur de l'Oise (1976-1978; voir Bernand, F. Reumaux, P. Reumaux), soit de mobilisation du groupe social comme l'affaire de Bruay (1972-1973; voir Caviglioli, Gavi) -, les inculpations judiciaires n'ont pas correspondu aux attentes fantasmatiques et rumorales et ont donc été accueillies avec scepticisme.

\section{Les autorités.}

Face à ce climat d'angoisse, les autorités ont jusqu'à présent adopté un profil bas. Quelques indices montrent cependant qu'elles y participent et en sont touchées. Tout décès inexpliqué d'enfant fait envisager le pire : on déclenche une enquête, une information judiciaire avec une célérité inhabituelle, et même avant l'autopsie l'on parle d'assassinat (cas d'Alexandre, 25 juillet 1989).

Par ailleurs, la justice fait des efforts de relations publiques pour signaler sa préoccupation : lors d'un meurtre récent, le ministre fait savoir qu'il a télégraphié aux procureurs généraux pour leur demander d'instruire cette affaire avec célérité (cas d'Aminata, 27 juillet 1989).

C'est que ce climat d'angoisse, cette conviction partagée et diffuse que l'on est dans une série contre laquelle l'autorité se révèle incapable d'agir, donne une résonance accrue aux mouvements activistes et entraîne des explosions de violence. Contestation de verdicts d'assises (Lons-le-Saunier, mai 1989), refus d'une reconstitution et attaque contre des avocats (Motte-au-Caire, juillet 1989), la liste n'est ni limitative ni close. Les autorités, face à de telles vagues, font rapidement figure d'accusés, de responsables. 


\section{Les activistes.}

Les mouvements activistes qui se développent dans le sillage de ces affaires comportent plusieurs couches et courants.

Comme dans le cas des disparitions, on y trouve tout d'abord les familles et les proches des victimes. De pareils faits éveillent une vive solidarité locale, et l'on notera que l'horreur semble grandir encore quand le meurtrier sadique se révèle être non un étranger ou un marginal, mais un membre jusqu'ici apparemment normal de la communauté touchée.

Leurs réactions sont archaïsantes - ainsi, la réclamation de la peine de mort est exprimée à ce niveau comme une véritable exigence de purification rituelle, de loi du talion.

Elles sont également populistes, et l'indignation devant l'incompétence, l'insensibilité, la cruauté des autorités - police et justice confondues, mais la révolte vise surtout la machine judiciaire - est un thème majeur, très mobilisateur.

Cependant, parents et voisins sont rapidement relayés par des professionnels, des militants qui exploitent et organisent ces tendances.

Le discours s'oriente vers la croisade : attaques contre la permissivité, l'homosexualité affichée, la pornographie - thèmes qui mobilisaient déjà ces militants. Ceux-ci affirment alors que les mœurs actuelles constituent des causes déterminantes de ces meurtres atroces.

L'appel à la peine de mort dérive alors vers l'action politique : il y a demande d'un référendum, action pour une modification législative et non plus exigence d'une catharsis. Le relais passe aux politiciens eux-mêmes, qui développent des thèmes sécuritaires et tentent d'exploiter ces mouvements pour leurs objectifs propres.

Un dernier aspect - le moins mobilisant émotionnellement de ces mouvements - est l'appel à la prévention : on rappelle aux parents la nécessité de la prudence, de la méfiance devant l'inconnu.

\section{Les médias.}

Comment les médias rendent-ils compte de ces événements traumatisants? Le type du média - quotidien ou magazine - est un facteur déterminant.

Dans la quasi-totalité des quotidiens, c'est une véritable stratégie participative qui est mise en cuvre, poussant le lecteur à s'identifier 
aux parents des victimes par divers procédés rhétoriques, dont l'établissement de listes situant le dernier assassinat dans le cadre d'une série angoissante par énumération de faits antérieurs analogues. Ces listes restent généralement muettes sur l'êventuelle élucidation du meurtre tout en indiquant le prénom, l'âge et les circonstances de l'assassinat: incertitude et angoisse sont alors renforcées.

Quand l'image peut jouer, son pouvoir évocateur sera largement mis à contribution aux mêmes fins participatives. Ainsi, dans ParisMatch de mars 1989, une double page de parents de victimes, portant chacun la photographie-portrait de l'enfant tué, précède une succession de photos du "temps du bonheur ", qu'accompagne un bref récit de chaque cas précisant si l'affaire a reçu ou non une solution. A côté de cette liste - qui fait huit pages - un texte de deux pages parle de la douleur des parents, de leurs révoltes et de leur rejet de la justice présentée comme indifférente ou même hostile; l'horreur des meurtres, les demandes de certaines associations pour une justice plus rigoureuse, plus efficace, mais aussi le refus de la vengeance par certains parents constituent des thèmes secondaires.

\section{CONCLUSION}

On le voit, ce sont des liens complexes qui se tissent entre le public, les activistes et les médias à l'occasion des disparitions et crimes. Rumeurs et fantasmes jouent un grand rôle dans l'élaboration des émotions et sont manipulés par les activistes qui tentent d'obtenir la mobilisation de la collectivité, de persuader le public qu'il y a là un problème social majeur. Pour les médias, rumeurs et fantasmes sont un des procédés permettant d'obtenir identification et participation du public. Cependant, ils sont à manipuler avec précaution, car leur usage inconsidéré entamerait la réputation du média. 


\section{BIBLIOGRAPHIE CITÉE}

Bennand (Carmen), " L'ombre du tueur. Réflexions anthropologiques sur une rumeur », Communications, 28, 1978, p. 165-185.

Besr (Joel), * Rhetoric in Claims-Making : Constructing the Missing Children Problem *, Social Problems, 34, 2, 1987, p. 101-121.

Best (Joel), * Missing Children, Misleading Statistics „, The Public Interest, 92, été 1988, p. 84-92.

Campion-Vincent (Véronique), * Complots et avertissements : les légendes urbaines *, Revue frangaise de sociologie, 30, 1989, p. 91-105.

Caniglou (François), « Bruay : sur la ville, l'ombre d'un doute „, Paris-Match, 1252, 5 mai 1973 , p. 43-45.

Gavı (Philippe), Bruay-en-Artois : seul un bourgeois aurait pu faire ca? ", Les Temps modernes, 312-313, juillet-août 1972, p. 155-260.

Kapferer (Jean-Noël), "Les disparitions de Mourmelon. Origine et interprétation des rumeurs *, Revue francaise de sociologie, 30, 1, 1989, p. 81-89.

Reumaux (Françoise), "L'aventure du héros de l'ombre \#, Cahiers de littérature orale, 15 , 1985, p. 61-80.

Reumaux (Patrick), L'Invité de Nogent, Paris, Grasset, 1977, 330 p.

Shibutani (Tamotsu), Improvised News. A Sociological Study of Rumor, New York, Indianapolis, The Bobbs-Merrill Company Inc., 1966, 262 p. 